\title{
Report
}

\section{Evaluation of a Sun Safety Education Program for High School Students in Beijing, China}

\author{
Dihui Lai, Jianchun Hao, Yu Fu, Shaowei Cheng \\ Department of Dermatology, Beijing Chuiyangliu Hospital, Beijing, China \\ Email address: \\ shorewave2008@139.com (Shaowei Cheng)
}

\section{To cite this article:}

Dihui Lai, Jianchun Hao, Yu Fu, Shaowei Cheng. Evaluation of a Sun Safety Education Program for High School Students in Beijing, China. Education Journal. Vol. 6, No. 2, 2017, pp. 94-99. doi: 10.11648/j.edu.20170602.14

Received: February 8, 2017; Accepted: March 9, 2017; Published: March 28, 2017

\begin{abstract}
Actions in reducing excessive sun exposure during childhood and adolescence has been a goal of a series of programs. The objective of this study was to evaluate a multi-component high school-based intervention program to increase sun safety knowledge and sun protection behaviors among students in Beijing, China. Volunteers from three high schools in Beijing were randomized to three groups. Group A was given a 2-year intervention program consisting of multi-unit presentations plus a series of educational materials. Group B was given a 1-year intervention program with educational materials only. Group C was served as a control group. Questionnaires were answered by the three groups at baseline, year 1, and year 2. In our study, group A achieved significant improvements in all six questions about the sun and sunscreen knowledge at year 1. Group B achieved significant improvements in two questions. Both groups A and B achieved significant improvements in avoiding sun exposure at noon and seeking shade. Group A achieved improvements in almost all sun knowledge and sun protection behaviors, with the exception of using sunglasses, at year 2 compared with year 1. We conclude that school-based sun protection intervention increases student knowledge and changes student sun protection behaviors. The 2-year program could achieved better results than the 1-year program.
\end{abstract}

Keywords: Sun Exposure, Sun Safety, Intervention, Adolescents

\section{Introduction}

It is commonly believed that excessive sun exposure is the main cause of melanoma and other types of skin cancers. Childhood and adolescence have been identified as key periods in the ethology of skin cancer $[1,2]$ given that more than $80 \%$ of sun exposure occurs during childhood [3]. Therefore, reducing excessive ultraviolet radiation (UVR) exposure during childhood and adolescence had been a goal of a series of programs. School is thought to be an effective venue for sun safety education. The U.S. Centers for Disease Control and Prevention recommends school programs on sun safety. Some school-based sun safety education programs have been reported in many studies, such as the "SunSmart Schools" in Victorian primary schools and other Australian states [4], a sun safety education program for primary school students in Switzerland [5, 6], raising sun protection and early detection awareness among Florida high schoolers [7], and the
SunWise Program in the US [8]. In Arizona, skin cancer prevention in the class education activity program, "Students are Sun Safe," was delivered by university students for middle and high school students [9].

In most school-based sun safety education programs, the following are included: a comprehensive sun safety policy; recommendations to use wide-brim hats, protective clothes, sunglasses, and an umbrella; proper application of sunscreen; and recommendations to avoid sun exposure at peak UVR times and seeking shade as much as possible [4].

Until now, most of the sun safety education programs have been conducted in Western countries. The majority of the studied populations have involved Caucasians. There has been little quantitative research on the long-term effects of sun protection education for school students in China. In the past decade, We have done some sun protection education for 
adults in China. Now we focus on childhood and adolescence sun safety education.

In the present study we implemented and assessed a multi-component high school-based intervention program to increase sun safety knowledge and sun protection behaviors among high school students in Beijing, China.

\section{Materials and Methods}

\subsection{Study Population}

Six hundred thirty-eight adolescents from three high schools in Beijing were recruited between May 2012 and May 2014. The students ranged in age between 12 and 18 years (mean, $14.4 \pm 2.5$ years), and included 315 boys $(49.3 \%)$ and 323 girls $(50.7 \%)$. Of the 638 students, 311 (48.7\%) were junior high school students and 327 (51.3\%) were senior high school students. Because students in grades 9 and 12 would graduate from junior and senior high school, respectively, and would not complete a 1-year follow-up evaluation, only students from grades 7,8,10,11 were recruited.

Participants were divided into three groups (intervention groups [A and B], and a non-intervention, which served as the control group [group C]) using a simple random number method. Group A included 103 boys (49.3\%) and 106 girls (50.7\%), ( mean age 14.3 \pm 2.1 years). Group B included 107 boys (49.1\%) and 111 girls (50.9\%) (mean age, $14.3 \pm 2.6$ years). And group C included 105 boys (49.8\%) and 106 girls (50.2\%), (mean age, $14.3 \pm 2.4$ years). To prevent loss of samples and to avoid the interactive effect among students within one class, random cluster sampling was adopted, designating a class as a unit.

\subsection{Study Design}

Students from group A received a 2-year multi-component intervention program (including two 45-min presentations each year with educational pamphlets). Students from group B received a 1-year program in which only educational pamphlets were provided.

All educational materials were designed by our research group, consisting of general UVR and sun safety knowledge, and some sun safety strategies specifically designed for Chinese adolescents. The following knowledge and behaviors were emphasized: (1) exposure to the sun is harmful in the morning, at nightfall, on cloudy days, and through windows; (2) sun protection behaviors, such as wearing wide-brimmed hats, long-sleeved clothes, and sunglasses, and holding up an umbrella, avoiding sun exposure at noon, and seeking shade, should be adopted as much as possible; (3) use a sufficient amount of sunscreen with a sun protection factor $(\mathrm{SPF})>30$ and protection grade of UVA (PA) $>++$ during sun exposure (applying every 2-4 h); and (4) use proper protective behavior according to the UV index every day.

Three self-administered, multiple-choice questionnaires were administered before and after the interventions. The questionnaire used in a previous sun safety study was used as a reference when preparing the questionnaires [10]. General instructions were provided by the same researcher before the survey that demonstrated how to fill out the questionnaire correctly.

The pre-intervention questionnaire consisted of three parts. The first part referred to general data, including name, gender, age, and information about skin photo type. The second part consisted of six questions aimed at evaluating knowledge of sun exposure and sunscreen. The third part consisted of seven questions aimed at identifying sun safety behaviors and two questions about the occurrence of UV-induced skin damage (sunburn and suntan) 1 year before the intervention.

The post-intervention questionnaires consisted of parts II and III of the pre-intervention questionnaire, the purpose of which was to identify changes in sun knowledge, sun safety behaviors, and the occurrence of UV-induced skin damages among students in the three groups.

\subsection{Sun Protection Education}

The study was performed in three stages.

\subsubsection{The First Stage}

In the first stage, a questionnaire was administered to all participants as the baseline in May 2012. After analyzing the initial results, a sun safety intervention program was designed to improve the sun knowledge and sun safety behavior for the intervention sub-groups.

\subsubsection{The Second Stage}

During the second stage, a series of sun safety education recommendations were provided to the intervention sub-groups. Students from group A received two 45-min presentations, which included the nature and dangers of UVR, methods people could use to protect themselves from UVR, and facts about and proper use of sunscreens. The skin photo type of each participant was identified during the presentation, and the participants were taught how to protect themselves from UVR according to the UV index and their skin photo types. Participants from group B received educational pamphlets containing the highlights of the presentations, which were also provided to the participants in group A. Students from group $\mathrm{C}$ did not receive any of the supplemental educational material, and thus served as the control group. Students from groups A and B received educational pamphlets twice during the following year. One year later, a second survey was distributed to all of the students from the three groups.

\subsubsection{The Third Stage}

In the third stage, students from group A received 2 additional 45-min sun protection educational classes and educational pamphlets during the 2 nd year, and received a $3 \mathrm{rd}$ survey on sun protection knowledge and the change in sun protection behavior at the end of year 2 .

\subsection{Statistical Analysis}

The results were quantitatively analyzed with SPSS 16.0 according to the intent-to-treat principle. The age data were 
normally distributed and described as the mean and standard deviation and analyzed using a t-test. The relationship between different groups was assessed by contingency tables using a $\chi^{2}$ test. For all tests, a $\mathrm{P}<0.05$ was considered statistically significant.

The study was reviewed and approved by the Ethics Committee of the Beijing Chuiyangliu Hospital.

\section{Results}

\subsection{Baseline}

At baseline, there was similar knowledge about sun exposure and sun screen, sun protection behaviors, and incidence of sunburn and suntan among students from all groups $(\mathrm{P}>0.05)$, with the exception of questions 2 and 4, in which there were statistical differences among groups (Tables 1-3).

Table 1. Awareness of ultraviolet (UV)-induced skin damage and knowledge of sunscreen before and after intervention.

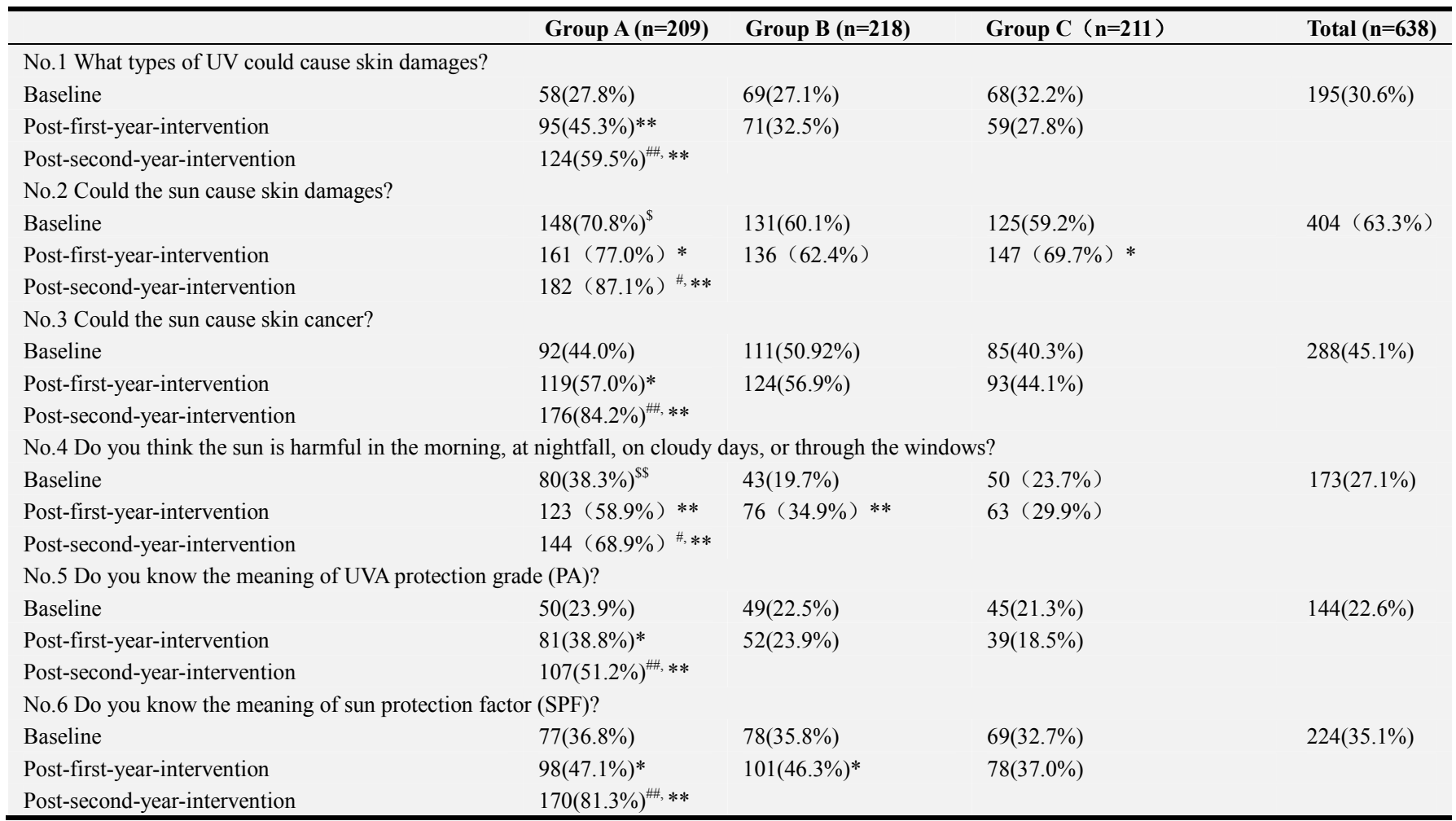

* Compare with the baseline $P<0.05$, ** Compare with the baseline $P<0.001$. ${ }^{\#}$ Compare with the post-first-year-intervention $P<0.05$, \# Compare with the post-first-year-intervention $P<0.001 .{ }^{\$}$ Compare with group $\mathrm{B}$ and $\mathrm{C} \mathrm{p}<0.05$. ${ }^{\$ \$}$ Compare with group $\mathrm{B}$ and $\mathrm{C} \mathrm{p}<0.001$

Table 2. Sun safety behavior before and and after intervention.

\begin{tabular}{|c|c|c|c|c|}
\hline & Group A $(n=209)$ & Group B $(n=218)$ & Group C $(n=211)$ & Total $(n=638)$ \\
\hline \multicolumn{5}{|l|}{ Sunscreen } \\
\hline Baseline & $113(54.1 \%)$ & $107(49.1 \%)$ & $95(45.0 \%)$ & $315(49.4 \%)$ \\
\hline Post-first-year-intervention & $112(53.6 \%)$ & $107(49.1 \%)$ & $87(41.2 \%)$ & \\
\hline Post-second-year-intervention & $170(81.3 \%)^{\# \#, * *}$ & & & \\
\hline \multicolumn{5}{|l|}{ Protective clothes } \\
\hline Baseline & $63(30.1 \%)$ & $56(25.7 \%)$ & $43(20.4 \%)$ & $162(25.4 \%)$ \\
\hline Post-first-year-intervention & $52(24.9 \%)$ & $57(26.1 \%)$ & $46(21.8 \%)$ & \\
\hline \multicolumn{5}{|l|}{ Hats } \\
\hline Baseline & $65(31.1 \%)$ & $55(25.2 \%)$ & $56(26.5 \%)$ & $176(27.6 \%)$ \\
\hline Post-first-year-intervention & $74(35.4 \%)$ & $68(31.2 \%)$ & $49(23.2 \%)$ & $191(29.9 \%)$ \\
\hline Post-second-year-intervention & $140(67.0 \%)^{\# \#, * *}$ & & & \\
\hline \multicolumn{5}{|l|}{ Sun umbrellas } \\
\hline Baseline & $68(32.5 \%)$ & $70(32.1 \%)$ & $60(28.4 \%)$ & $198(31.0 \%)$ \\
\hline Post-first-year-intervention & $70(33.5 \%)$ & $62(28.4 \%)$ & $54(25.6 \%)$ & $186(29.2 \%)$ \\
\hline Post-second-year-intervention & $133(63.6 \%)^{\# \# * *}$ & & & \\
\hline Baseline & $68(32.6 \%)$ & $42(19.3 \%)$ & $45(21.3 \%)$ & $155(24.3 \%)$ \\
\hline Post-first-year-intervention & $55(26.3 \%)$ & $49(22.5 \%)$ & $35(16.6 \%)$ & \\
\hline Post-second-year-intervention & $42(20.1 \%)$ & & & \\
\hline Avoiding sun exposure at noon & & & & \\
\hline
\end{tabular}




\begin{tabular}{lllll}
\hline & Group A $(\mathbf{n}=\mathbf{2 0 9})$ & Group B $(\mathbf{n}=\mathbf{2 1 8})$ & Group C $(\mathbf{n}=\mathbf{2 1 1})$ & Total $(\mathbf{n = 6 3 8})$ \\
\hline Baseline & $39(18.7 \%)$ & $42(19.3 \%)$ & $50(23.7 \%)$ & $131(20.5 \%)$ \\
Post-first-year-intervention & $74(35.4 \%)^{* *}$ & $68(31.2 \%)^{*}$ & $49(23.2 \%)$ & \\
Post-second-year-intervention & $140(67.0 \%)^{\# * * *}$ & & & \\
Seeking shade as far as possible & & & \\
Baseline & $58(27.8 \%)$ & $51(23.4 \%)$ & $60(28.4 \%)$ & $169(26.5 \%)$ \\
Post-first-year-intervention & $80(38.3 \%)^{*}$ & $75(34.4 \%)^{*}$ & $54(25.6 \%)$ & \\
Post-second-year-intervention & $133(63.6 \%)^{\# * * *}$ & & & \\
\hline
\end{tabular}

* Compare with the baseline $P<0.05$, ** Compare with the baseline $P<0.001,{ }^{\#}$ Compare with the post-first-year-intervention $P<0.05$, \# Compare with the post-first-year-intervention $P<0.001$.

Table 3. Occurrence of UV-induced skin damage before and after intervention

\begin{tabular}{|c|c|c|c|c|}
\hline & Group A (n=209) & Group B $(\mathbf{n}=\mathbf{2 1 8})$ & Group C $(n=211)$ & Total $(n=638)$ \\
\hline \multicolumn{5}{|c|}{ Do you had ever suffered from sunburn in resent 1 year } \\
\hline Baseline & $101(48.3 \%)$ & $92(42.2 \%)$ & $69(32.7 \%)$ & $262(41.1 \%)$ \\
\hline Post-first-year-intervention & $63(30.1 \%)^{* *}$ & $68(31.2 \%)^{*}$ & $60(28.4 \%)$ & \\
\hline Post-second-year-intervention & $33(15.8 \%)^{\# \#, * *}$ & & & \\
\hline \multicolumn{5}{|c|}{ Do you had ever gotten suntan in resent 1 year } \\
\hline Baseline & $165(78.9 \%)$ & $168(77.1 \%)$ & $157(74.4 \%)$ & $490(76.8 \%)$ \\
\hline Post-first-year-intervention & $123(58.9 \%)^{* *}$ & $136(62.4 \%) *$ & $142(67.3 \%)$ & \\
\hline Post-second-year-intervention & $89(42.6 \%)^{\#, * *}$ & & & \\
\hline
\end{tabular}

* Compare with the baseline $P<0.05, * *$ Compare with the baseline $P<0.001 .{ }^{\#}$ Compare with the post-first-year-intervention $P<0.05$, ${ }^{\# \#}$ Compare with the post-first-year-intervention $P<0.001$.

\subsection{First Follow-Up}

All of students answered the questionnaires 1 year later. The group A students achieved significant improvements in all six questions involving knowledge of $\mathrm{UV}$ and sunscreen $(\mathrm{P}<0.05$ or $\mathrm{P}<0.001)$. The group $\mathrm{B}$ students achieved significant improvements in two questions $(\mathrm{P}<0.05$ or $\mathrm{P}<0.001)$. The group $\mathrm{C}$ students showed improvement in one question $(\mathrm{P}<$ 0.05; Table 1).

For questions involving sun protection behaviors, students from groups A and B achieved significant improvements in avoiding sun exposure at noon and seeking shade $(\mathrm{P}<0.05$ or $\mathrm{P}<0.001)$. Students from groups $\mathrm{A}$ and $\mathrm{B}$ did not achieve improvements in the use of sunscreen, protective clothing, hats, sun umbrellas, or sunglasses $(\mathrm{P}>0.05)$. Students from group $\mathrm{C}$ did not demonstrate improvement in any of the aforementioned items $(\mathrm{P}>0.05$; Table 2$)$.

We also queried students who had a sunburn or suntan in the past year. Students from groups A and B achieved a significant decrease in the incidence of sunburns and suntans in the past year $(\mathrm{P}<0.05$ or $\mathrm{P}<0.001)$, while students from group $\mathrm{C}$ had the same incidence of sunburns and suntans in the past year compared with baseline ( $\mathrm{P}>0.05$; Table 3$)$.

\subsection{Second Follow-Up}

Students from group A answered questionnaires 2 years later. They achieved significant improvements in all six questions involving UV and sunscreen knowledge compared with the first follow-up $(\mathrm{P}<0.05$ or $\mathrm{P}<0.001)$ and the baseline $(\mathrm{P}<0.001$; Table 1$)$.

For questions involving sun protection behavior, students from group A achieved improvements in six of seven questions compared with the first follow-up and baseline $(\mathrm{P}<0.001)$, with the exception of using sunglasses $(P>0.05$; Table 2$)$.

Students from group A also achieved a significant decrease in the incidence of sunburns $(\mathrm{P}<0.001$ compared with year 1 and baseline $)$ and suntans $(\mathrm{P}<0.05$ compared with year 1 , and $\mathrm{P}<0.001$ compared with baseline) during year 2 (Table 3 ).

\section{Discussion}

A number of studies $[5,8,9,11,12]$ have attempted to change the sun protection behaviors of adolescents by offering sun safety education programs in schools. Most of the school-based interventions increase the sun protection knowledge, while only a limited number of interventions changed intended or actual behaviors. For example, in the "Living with the Sun" (LWS) program, the LWS group applied sunscreen more frequently than the control group, and were more likely to wear a hat (72\% vs. $59 \%)$ and use a sun umbrella (75\% vs. 64\%) [11]. Most of the studies have reported an increase in sun safety knowledge without an improvement in intention or actual behavior [7, 12].

The gap between sun safety knowledge/attitude and behavior was the focus of our previous study [10] and other studies $[5,9]$. It is generally believed that it is easier to increase sun safety knowledge than to change intended or actual sun safety behavior. Reinau et al. [5] reported that after an intervention, sun safety knowledge increased significantly, but there was no change in sun-protective behavior. In Arizona, after exposure to the "Students are Sun Safe" project, participants answered knowledge-based questions correctly; however, there were minimal differences in self-reported sun-safety behaviors, although the participants did report an intent to change [9]. In agreement with those studies [5, 9], the students in our study exhibited enormous improvement in sun knowledge; however, we failed to elicit behavioral change in most items at year 1. The students in groups A and B achieved progress in avoiding sun exposure and seeking shade at noon, but neither of the groups changed their use of sunscreens, hats, 
protective clothing, sunglasses, and sun umbrellas. This finding contradicted the finding reported by Reinau [5, 6], in which $>95 \%$ of students benefited from the protection of sunscreen, but only $36 \%$ sought shade on sunny days.

A number of studies [13] have indicated that the choice to use sun protection is likely to involve psychosocial factors. The sun safety behavior of adolescents may depend on the situation and motivational factors, such as school policy, and peer and friendship groups [13]. In China, sunscreen is commonly believed to be a type of cosmetic which should only be used by girls and ladies. Using a sun umbrella is also thought to be appropriate only for girls and ladies. Using sunglasses is commonly believed to be a bad behavior, and thus is forbidden in many schools. These prejudices have hindered improvements in sun safety behaviors. After identifying these prejudices, we emphasized the importance of using sunscreen, sun umbrellas, and sunglasses as sun safety behavior in the year 2 intervention.

Buller et al. [14] reviewed a series of sun protection programs and interventions in North America. They reported that a single $30-60 \mathrm{~min}$ presentation alone is insufficient to improve the sun protection behavior in children. Therefore, multi-unit presentations are suggested, including several days of presentations that include a series of instructional materials related to sun safety. Girgis et al. [15] showed that students in the intensive intervention group had significant improvement in sun safety behavior compared to the control and standard (minimal) intervention groups. There was no difference in the level of protection between the control and standard intervention groups, indicating that minimal intervention was not effective in changing the sun safety behavior of children. Our study showed that students from group A had significant improvement in all six questions involving sun and sunscreen knowledge, while group B achieved improvement in only two questions. This finding suggests that sun protection intervention by written materials alone is insufficient to improve the sun protection knowledge and sun protection behavior amongst students. Multi-unit presentations plus a series of educational materials could yield better results.

Another question is whether or not the education program should last greater than one year. Previous studies have shown that the key points of a successful program may be the intensity and duration of the intervention. Interventions relying on a single session have not been shown to be successful at modifying sun protection behaviors at school [16]. Indeed, interventions over the course of the school year have proven more successful [17]. Olson et al. [18] assessed the impact of 1 and 2 years of intervention, and the results showed that a significant effect occurred after 2 years, but not after 1 year of exposure to the intervention. Norman et al. [19] reported that at year 2 more adolescents in the intervention group had moved to the action or the maintenance stage of change than the control group (25.1\% vs. 14.9\%). Sun protection behavior was also shown to be positively associated with the completion of more intervention sessions.

Even though we failed to change the habits of students in use of sunscreen, protective clothing, hats, sun umbrellas, and sunglasses in the first year, at the end of year 2, with persistent improvement in sun safety knowledge, we successfully achieved improvements for students in using sunscreen, protective clothing, hats, and sun umbrellas, and more improvements in avoiding sun exposure and seeking shade at noon. We speculate that an everlasting intervention would continually increase sun knowledge amongst students and increase awareness, which is an important precursor to achieving behavioral change if the duration of the intervention is long enough.

The limitations of the current study include the lack of a control group at year 2 and differences in some questions among groups at baseline. In China, most junior and senior high schools are two independent schools. Most students from grade 9 will change schools when they graduate from junior high school. Therefore, we have to select students from grades 7 and 10 as group A to complete the 2-year intervention. Students from grades 8 and 11, who would graduate 1 year later, were selected as group B and the control group. The difference in grade selection might cause differences in some questions among the groups at baseline. We do not believe these differences influenced our results because we compared improvements within the same group (not among groups).

\section{Conclusion}

In conclusion, we conclude that a school-based sun protection intervention increased sun protection knowledge of students, and to a certain extent, changed some behaviors at year 1. With a persistent improvement in sun safety knowledge, most sun protection behaviors improved greatly if the duration of the intervention was long enough. Multi-unit presentations plus a series of educational materials could achieve better results than educational materials alone.

\section{Acknowledgements}

This project was funded by a grant from the Social Development Project 2012 of Chaoyang Beijing. Project No: SF1113.

\section{References}

[1] Whiteman DC, Whiteman CA, Green AC. Childhood Sun Exposure as a Risk Factor for Melanoma: A Systematic Review of Epidemiologic Studies. Cancer Causes Control 2001: 12: 69-82.

[2] Corona R, Dogliotti E, D'Errico M, et al. Risk Factors for Basal Cell Carcinoma in a Mediterranean Population: Role of Recreational Sun Exposure Early in Life. Arch Dermatol 2001: 137: 1162-1168.

[3] Truhan AP. Sun protection in childhood. Clin Pediatr 1991: 30: 676-681.

[4] Turner D, Harrison SL, Buettner P, Nowak M. School sun-protection policies-does being SunSmart make a difference? Health Educ Res 2014: 29: 367-377. 
[5] Reinau D, Meier CR, Gerber N, Surber C. Evaluation of a sun safety education programme for primary school students in Switzerland. Eur J. Cancer Prev 2014: 23: 303-309.

[6] Reinau D, Meier C, Gerber N, Hofbauer GF, Surber C. Sun protective behaviour of primary and secondary school students in North-Western Switzerland. Swiss Med Wkly 2012: 142: w13520.

[7] Geller AC, Shamban J, O'Riordan DL, Slygh C, Kinney JP, Rosenberg S. Raising sun protection and early detection awareness among Florida high schoolers. Pediatr Dermatol 2005: 22:112-118.

[8] Emmons KM, Geller AC, Viswanath V, et al. The SunWise Policy intervention for school-based sun protection: a pilot study. J. Sch Nurs 2008: 24: 215-221.

[9] Davis R, Loescher LJ, Rogers J, et al. Evaluation of Project Students are Sun Safe (SASS): A. University Student-Delivered Skin Cancer Prevention Program for Schools. J. Cancer Educ 2015: 30: 736-742.

[10] Cheng S, Guan X, Cao M, Liu Y, Zhai S. Randomized trial of the impact of a sun safety program on volunteers in outdoor venues. Photodermatol Photoimmunol Photomed 2011: 27: 75-80.

[11] Sancho-Garnier H, Pereira B, Césarini P. A. cluster randomized trial to evaluate a health education programme "Living with Sun at School". Int J. Environ Res Public Health 2012: 9: 2345-2361.

[12] Dadlani C, Orlow SJ. Planning for a brighter future: A. review of sun protection and barriers to behavioral change in children and adolescents. Dermatol Online J 2008 Sep 15: 14: 1 .
[13] Hawkes AL, Hamilton K, White KM, McD Young R. A. randomised controlled trial of a theory-based intervention to improve sun protective behaviour in adolescents ('you can still be HOT in the shade'): study protocol. BMC Cancer 2012 Jan 3: 12: 1 .

[14] Buller DB, Borland R. Skin cancer prevention for children: A. critical review. Health Educ Behavior 1999: 26: 317-341.

[15] Girgis A, Sanson-Fisher RW, Tripodi DA, Golding T. Evaluation of interventions to improve solar protection in primary schools. Health Educ Q. 1993: 20: 275-287.

[16] Buller DB, Taylor AM, Buller MK, Powers PJ, Maloy JA, Beach BH. Evaluation of the sunny days, healthy ways sun safety curriculum for children in kindergarten through fifth grade. Pediatr Dermatol 2006: 23: 321-329.

[17] Hunter S1, Love-Jackson K, Abdulla R, et al. Sun protection at elementary schools: a cluster randomized trial. J. Natl Cancer Inst 2010: 102: 484-492.

[18] Olson AL, Gaffney C, Starr P, Gibson JJ, Cole BF, Dietrich AJ. SunSafe in the Middle School Years: a community-wide intervention to change early-adolescent sun protection. Pediatrics 2007: 119: e247-256.

[19] Norman GJ1, Adams MA, Calfas KJ, et al. A randomized trial of a multicomponent intervention for adolescent sun protection behaviors. Arch Pediatr Adolesc Med 2007: 161: 146-152. 